\title{
Dynamic Finite Element Model Updating for On-load Tap Changer based on Super-model
}

\author{
Zhiyuan Liu ${ }^{1}$, Hongseng Zou ${ }^{1}, H u i \mathrm{Miao}^{2}$, Dapeng $\mathrm{Chen}^{3}$ and Lin Guo ${ }^{4}$ \\ ${ }^{1}$ State Grid Ningxia Electric Power Co. Ltd. Maintenance Company, 750000Yinchuan, China \\ ${ }^{2}$ College of Energy and Power Engineering, Nanjing University of Aeronautics and Astronautics, 210016Nanjing, China \\ ${ }^{3}$ Nanjing Unitech Electric Power Co. Ltd, 211100Nanjing, China \\ ${ }^{4}$ State Grid Chongqing Electric Power Company, 400015Chongqing, China
}

\begin{abstract}
A method is presented for dynamic model updating of on-load tap changer (OLTC). Based on a sensitivity-based optimization method, the initial simplified finite element (FE) model of OLTC component is updated using the analytical results of the FE super-model. The objective of model updating is to reduce the frequency difference between the simplified FE model and the super-model, and to make the simplified model accurately represent dynamic characteristics of the super-model. The updated simplified models can be further used in the modeling and analysis of the whole OLTC model. The results, taking the base of OLTC as example, indicate that the dynamic behavior of the updated simplified model match well with that of the super-model. Subsequently, the dynamic behavior of OLTC assembled with the updated parts is further predicted by modal analysis. The presented method improves the calculation efficiency, as well as accuracy, which has broad application prospects for dynamic prediction of complex structures in engineering.
\end{abstract}

\section{Introduction}

An on-load tap-changer (OLTC) is a mechanical subassembly of a converter transformer for on-load voltage regulating[1]. During the operation, OLTC is subject to a lot of mechanical impacts and electric shocks, which may lead to defects in a tap changer, such as contact wear, springs weakening or broken, fastener loosening or falling off, contact degradation, blocked half-way[2]. With the increasing application of the converter transformer in the national grid, the incidence of OLTC faults also increases and the problems of the grid safety stand out day by day. In china, according to statistics, OLTC failures account for about $40 \%$ of the transformer faults, while most of the faults of the tap changer are caused by mechanical failure[3]. Therefore, it is necessary to study the mechanical faults to improve the stability and reliability of OLTC.

During the frequent operation of OLTC, the mechanical vibration caused by the collision or friction between the mechanical parts is the main cause of the mechanical failure of OLTC. At present, the research on vibration faults mechanism of OLTC mainly includes experimental research and theoretical study. In the aspect of experimental study, Cichon et al.[4] present their research works considering selection of measuring transducer applied for registration of acoustic emission signals generated by on-load tap changers. Seo et al.[5] present the set-up of OLTC vibration measurement system, and based on test data, base waveform determination and waveform alignment methods are proposed for comparing waveforms of different tap positions. In addition, they introduce savitzky-Golay filter to process the signals acquired from a joint vibroacoustic and arcing measurement system[6]. This method can improve the visibility of OLTC's mechanical operation for an effective online condition monitoring. With respect to theoretical research, Kang et al.[7] present an OLTC vibration signal characterization technique using the continuous wavelet transform (CTW), and the essential features from the original signals are extracted to provide reliable indications of the health of the equipment. Mikheev et al.[8] propose a new method of oscillography to evaluate the contactor parameters of SDV-type on-load tap changers with opened tank and partial removal of transformer oil. Li et al.[9] investigate a novel strategy based on a Hidden Markov Model (HMM) for mechanical fault diagnosis of OLTCs, and the failure feature vectors are extracted for constructing a discrete spectrum vector of the vibration signals. However, from the references about theoretical study, analytical models are established based on the experimental data of OLTC, so they are dependent on a large amount of experiments, which cost users too much effort. With the development of the computer technology, the fault diagnosis method based on finite element model is becoming widely used in industrial. As for complex OLTC structure, the FE model can be more easily obtained by finite element method compared with the traditional analytical modeling method. However, the 
finite element model often has a significant discrepancy in the process of modeling since the model is often influenced by some possible sources[10]. Therefore, how to establish an accurate OLTC FE model is really a critical problem demanding prompt solution.

Based on the model updating theory, this paper studies the dynamic modeling method of OLTC, and puts forward a dynamic modeling and updating method based on the super-model. The purpose of this paper is to establish a simplified FE model for dynamic analysis of the whole OLTC, so that the compution accuracy and efficiency could be simultaneously assured in the dynamic analysis of the whole OLTC model. Taking dynamic modeling of OLTC base as an example, the simplified model of the base is updated using the model updating method based on the dynamic simulation results of its super-model. Then, the rationality and effectiveness of the modeling method is further verified. Finally, the dynamic characteristics of the assembled OLTC is also predicted based on the updated parts of OLTC.

\section{Model updating based on super-model}

\subsection{Model updating procedure}

As the discretization and idealization of the actual structure, the finite element model often has a significant discrepancy in the process of modeling since the model precision is often influenced by some uncertain factors, such as element type, material property, mesh quality, manufacturing tolerance and boundary condition. Therefore, the method of model updating should be carried out to improve the accuracy of the model in the sense that the deviations between test and analysis are minimized. However, at the beginning of the design, the test data can't be obtained due to the absence of the physical prototype. When we perform the prediction of the design model, the computation efficiency is also very low if the whole FE model of OLTC is established without any simplification. In view of this problem, we can establish a simplified model instead of the whole complex model in the prediction. Based on model updating theory, the simplified model can be obtained by the simulation data of the super-model instead of test model.

The purpose of dynamic model updating based on super-model is to improve the accuracy of the simulation model by using the simulation data obtained from the super-model, so that the updated model can accurately reflect the dynamic characteristics of the structure in the required precision range, and it can be further applied to the structural optimization design, the dynamic response prediction, and the fault diagnosis. Figure 1 shows the procedure of structural dynamics model updating based on super-model[11]. In the process of model updating, the initial finite element model of the geometric model is updated using the dynamic parameters of the supermodel. After updating, the FE model is able to produce natural frequencies and mode shapes in close agreement with that of the super-model.
Generally, the model updating procedure based on super-model includes modeling and analysis of initial FE simplified model and super-model, verification of the prediction data of the initial FE model, correlation analysis, model updating, and evaluation of the updated model, in order to reduce the error between the prediction results of the simplified FE model and the super-model, and finally obtain the validated FE model.

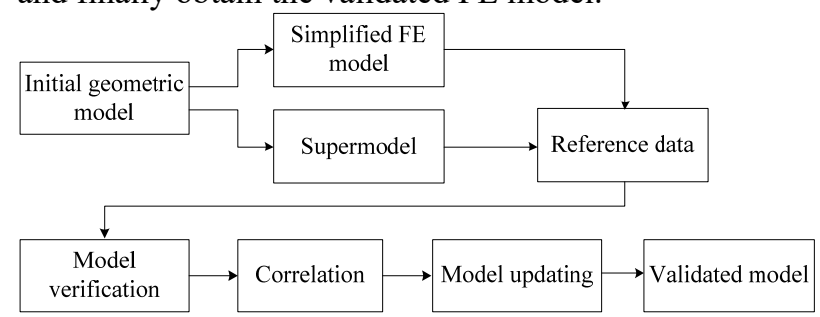

Fig. 1. Model updating procedure.

\subsection{Modeling of super-model}

The traditional dynamic modeling mostly relies on the designer's experience. Usually, an reasonable design model can be obtained through a lot of trial, which costs too much. The super-model[12] is a reasonable threedimensional finite element model with very high degree of freedom. The super-model has sufficient precision to reflect the physical characteristics of the actual structure. It not only completely describes the geometric features of the structure, but also accurately reflects the dynamic characteristics such as natural frequencies and mode shapes. The super-model can be used as a reference model for model updating and validation of the design model in the structural design stage, which greatly improves the design accuracy and efficiency and reduce the design cost.

In order to obtain the super-model accurately and efficiently, a method suitable for the establishment of the super-model needs to be developed. The principle of modeling for the super-model is mainly considered from two aspects. First, the model accuracy must be high. It is the most important index to measure the quality of the super-model which should be able to accurately reflect the dynamic characteristics of the geometric model and the simulation data of the super-model represents the expected result of the design. Secondly, on the premise of guarantee the model accuracy, the calculaiton scale of the super-model should be minimized to improve the simulation efficiency.

For a single part of the mechanical strcuture, the modeling process for the the super-model can be performed as follows:

(1) Clean of geometry

Check the geometric model and eliminate the errors in the modeling process as well as the compatibility issues between the geometric modeling software (e.g. UG) and finite element analysis software (e.g. ANSYS) to ensure the geometric model satisfying the design expectations so that meshing can be done;

(2) Analysis of initial FE model

The geometric model is meshed using second-order tetrahedral elements with a coarse mesh size, and modal 
analysis is performed under the free-free boundary conditions within the required frequency range;

(3) Iterative Analysis of mesh model

Mesh size will be gradually refined through iterative analysis. Correlation analysis is performed between the current refined mesh model and the previous reference model. The modal pairs of the same vibration modes are determined, and then frequency differences are calculated.

(4) Convergence analysis of the model

Check the convergence of the modal results according to the convergence criteria of the model. If the results are not convergent, refinement continues, otherwise, stops. The average frequency error and maximum frequency error of the current model and the reference model are used to measure the convergence of the mesh. These two errors can be set according to the requirements of the user. To quantitatively describe the convergence index of the model, the average frequency error and the maximum frequency error of the current calculation model can be expressed as

$$
\begin{gathered}
\eta=\frac{1}{n} \sum_{j=1}^{n}\left|f_{i, j}-f_{i+1, j}\right| \\
\delta=\max \left|f_{i, j}-f_{i+1, j}\right|, j=1,2, \ldots, n
\end{gathered}
$$

Where $f_{i, j}$ represents the $j$ th modal frequency of the model calculated at the $i$ th iteration, and $n$ is the modal order.

(5) Determine the super-model

According to the convergence index of the supermodel, the appropriate mesh size is determined and the geogmetric model is remeshed, and correlation analysis of the remeshed model is carry out with the previous meshed model. The super-model is obtained by satisfying the requirement of convergence index.

\subsection{Model updating and correlation}

The main purpose of model updating based on supermodel is to adjust parameters in the FE model to minimize the errors between the analytical model and super-model in order that the predictions of the dynamic characteristics from the FE model match the data from the super-model in the frequency range of interest. Model updating is one of the most important steps in the model validation. In the process of model updating, the variations of the parameters in the predicted model must satisfy the constraint conditions of the practical physical structure. Accordingly, the model updating problem is essentially an optimization, which can be expressed as

$$
\begin{cases}\min & g(\boldsymbol{x})=\|\boldsymbol{W R}(\boldsymbol{x})\|_{2}^{2}=\left\|\boldsymbol{W}\left(\boldsymbol{f}_{r}-\boldsymbol{f}_{a}(\boldsymbol{x})\right)\right\|_{2}^{2} \\ \text { s.t. } & \boldsymbol{x}^{L} \leq \boldsymbol{x} \leq \boldsymbol{x}^{U} \\ & \boldsymbol{f}_{a}^{L}(\boldsymbol{x}) \leq \boldsymbol{f}_{a}(\boldsymbol{x}) \leq \boldsymbol{f}_{a}^{U}(\boldsymbol{x})\end{cases}
$$

Where $g(x)$ is the objective function, $\boldsymbol{W}$ is the weighting matrix, and $\boldsymbol{R}$ is the residual vector that can be expressed as $\boldsymbol{R}(\boldsymbol{x})=\boldsymbol{f}_{\boldsymbol{r}}-\boldsymbol{f}_{a}(\boldsymbol{x})$ in which $\boldsymbol{f}_{r}$ and $\boldsymbol{f}_{a}$ respectively denote vectors of the reference and predicted dynamic properties. The vector expressed as $\boldsymbol{x}=\left[\boldsymbol{x}_{1}, \boldsymbol{x}_{2}, \boldsymbol{x}_{3}, \ldots, \boldsymbol{x}_{n}\right]^{\mathrm{T}}$ represents the design variables, and each variable has been specified upper and lower bounds. The model updating problem can be solved by the sensitivity-based optimization method[13].

Usually, the modal parameter $\boldsymbol{f}_{\boldsymbol{a}}(\boldsymbol{x})$ is nonlinear functions of the parameter $\boldsymbol{x}$ to be updated. To transform the nonlinear problem to linear form, the functional relationship between the reference and the initial analytical results can be approximated by a first-order Taylor-series expansion with respect to the structural parameters as follows

$$
f_{r}=f_{a}(x)+S \Delta x+o(\Delta x)
$$

where $\boldsymbol{S}$ is the sensitivity matrix in relation to updating parameters evaluated at the initial estimate $\boldsymbol{x}_{a}$ or, in the following iterative scheme, at the current parameter estimate $\boldsymbol{x}_{\boldsymbol{c}}$. So $\Delta \boldsymbol{x}=\boldsymbol{x}_{\boldsymbol{c}}-\boldsymbol{x}_{\boldsymbol{a}}$. The higher-order terms of the Taylor-series expansion are neglected due to the changes in the updating parameters between successive iterations are very small. The equation (4) has the form of

$$
S \Delta \boldsymbol{x}=\boldsymbol{R}
$$

Where $\boldsymbol{R}=\boldsymbol{f}_{r}-\boldsymbol{f}_{a}(\boldsymbol{x})$, is the residual vector. It is obvious that if the equation (5) is over-determined, a solution can be found. However, the matrix $\boldsymbol{S}$ may be singular if not all the reference data are independent, which can be done by calculating a generalized inverse for $\boldsymbol{S}$. Then we have the form

$$
\Delta \boldsymbol{x}=\boldsymbol{S}^{+} \boldsymbol{R}
$$

Where $\boldsymbol{S}^{+}$is the generalized inverse for $\boldsymbol{S}$. The solutions with respect to correction factor $\Delta \boldsymbol{x}$ can be solved by the least-squared method.

In the process of model updating, the Modal Assurance Criterion (MAC)[14] is often used to measure the similarity of the mode shapes between the analytical and reference models. The MAC is defined as

$$
\operatorname{MAC}\left(\boldsymbol{\phi}_{r, i}, \boldsymbol{\phi}_{a, j}\right)=\frac{\left|\boldsymbol{\phi}_{r, i}^{\mathrm{T}} \boldsymbol{\phi}_{a, j}\right|^{2}}{\left(\boldsymbol{\phi}_{r, i}^{\mathrm{T}} \boldsymbol{\phi}_{r, i}\right)\left(\boldsymbol{\phi}_{a, j}^{\mathrm{T}} \boldsymbol{\phi}_{a, j}\right)}
$$

Where $\phi_{r, i}$ is the $i$ th reference mode shape, and $\phi_{a, j}$ is the $j$ th analytical mode shape. The MAC value is between 0 and 1. Two mode shapes with a MAC value of 1 indicates identical modes, here the reference model is refer to the super-model.

\section{Modeling and updating of simplified model}

\subsection{Super-model}

OLTC is of complex structure, and consists of a large number of components such as bases, connecting rods, insulating plates, hanging plates, transition resistors and active and static contacts. All components are connected by fasteners such as bolts, screws, and pins. As shown in Figure 2, taking the super-model modeling of the OLTC base as an example, the super-model of the base is established by the super-model modeling method in section 2.2, and the base is created by the second order tetrahedron element (10 nodes) under free-free boundary condition. The first 10 modes within the modal frequency range of $2000 \mathrm{~Hz}$ are analyzed. The FE mesh of the super- 
model is dense, so the super-model can be meshed by iterating the uniform mesh size. With no consideration of local mesh refining, the initial mesh size is set as $10 \mathrm{~mm}$, and the iteration step increment is $-1 \mathrm{~mm}$, finally the mesh is gradually refined to $1 \mathrm{~mm}$. The average frequency error is set to $0.3 \%$, the maximum frequency error is set to $0.5 \%$, and all the modal correlation coefficients of the matched modal pair are greater than 0.95 . As shown in Figure 3, the super-model is obtained by iteratively changing mesh size. The optimal mesh size of the supermodel is $5 \mathrm{~mm}$, and the degree of freedom is 979248 . The elastic modulus of the base is $210 \mathrm{GPa}$, and the Poisson's ratio is 0.3 , and the density is $7850 \mathrm{~kg} / \mathrm{m}^{3}$. Figure 4 shows the first 10 modal modes of the supermodel of the base.

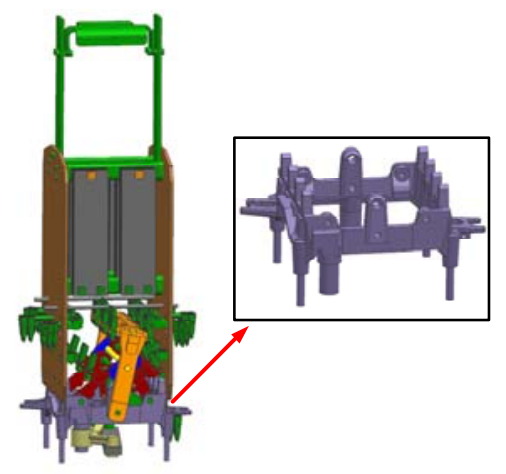

Fig. 2. The base of OLTC.

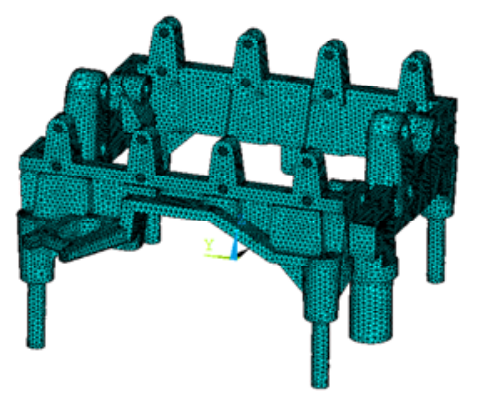

Fig. 3. Super-model of the base.
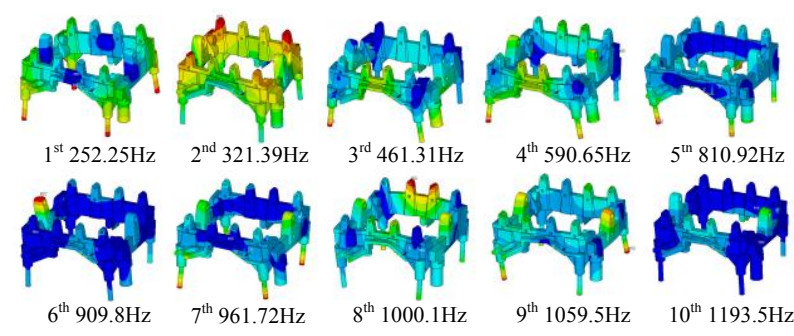

Fig. 4. Mode shapes of the base.

\subsection{FE model simplification}

The super-model has high precision and can be used for the dynamic analysis of the whole OLTC model. However, the calculation scale is especially large for the super-model calculation. It takes a lot of computation resource and the computational efficiency is extremely low. However, the calculation efficiency and accuracy of dynamic analysis of OLTC can be greatly imporved using the simplified model instead of the super-model.

(1) Simplification of geometric structure

Some small-size geometric features such as smalldiameter holes, chamfering and rounding can be removed. Because they have little effect on the dynamic characteristics of the structure, but drastically increases the number of local degrees of freedom of the model, which seriously affects the computational efficiency of the finite element model. In the process of establishing the simplified model, as the mesh size becomes larger, the mesh quality of the chamfer portion deteriorates sharply, which makes the local mesh division become difficult. Therefore the generation of deformed meshes can be reduced by removing the chamfer and the mesh of the geometrical structure may become smoother. For some of the boss structures, removal can be made depending on the situation, when the bosses with small size, or they only affect the local modes of the structure. The initial geometric model of the base is shown in Figure 5(a), and the simplified geometric model is shown in Figure 5(b).

(2) Simplification of mesh grids

A coarser mesh size is chosen as the overall mesh size of a simplified model compared with the mesh size of the super-model. For some geometric structures of small size, mesh size can be adjusted appropriately to reduce the generation of deformed meshes. After obtaining a simplified geometry model, the structure can be partitioned according to geometric features to obtain a simplified model conveniently by controlling the mesh size of different geometric features, as shown in Figure 6(a). Then, the geometric model of each block is meshed, and the continuity of the mesh is maintained between adjacent block structures. The calculation scale of the simplified model can be controlled by adjusting the mesh size of the block structure, or by referring to the modeling method of super-model. The second method controls the computational scale of the simplified model by controlling the convergence condition of the dynamic parameters of the model. Here, the simplified model can be obtained by adjusting its mesh size with a degree of freedom (DOF) of $1 / 10$ of super-model, as shown in Figure $6(b)$, and the DOF of the simplified model is 109647.

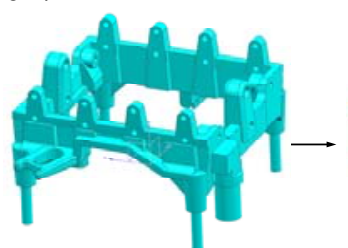

(a)

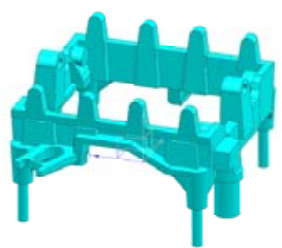

(b)
Fig. 5. Geometric simplification for the base.

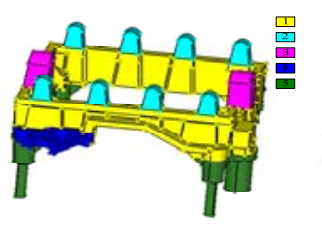

(a)

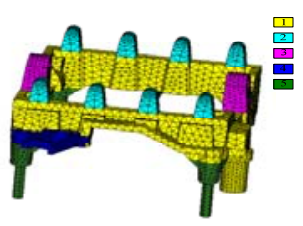

(b)
Fig. 6. Finite element meshing for the base. 
Table 1 gives the comparison of frequencies and MAC between the simplified model and super-model. From Table 1, the maximum frequency error between the simplified model and the super-model is $3.91 \%$. The average frequency error of the simplified model and the super-model is $2.46 \%$. The MAC values of the simplified model and the super-model are all above 0.9, and most of the matching modal pairs have high correlation of mode shapes. The results show that the model simplification method is reasonable. Although the simplified mass and stiffness distribution of the model and the discrete error of the mesh give rise to some errors of modal parameters between the simplified model and the super-model, they are basically within an acceptable range. Table 2 gives the comparison of calculation scale between the simplified model and the super-model. It can be seen from table 2 that the DOF and calculation time of the simplified model are reduced to $11.2 \%$ and $10.34 \%$ of that of the super-model, respectively. So the computational efficiency is greatly improved after model simplification.

Table 1. Comparison of frequencies and MAC between the simplified model and super-model.

\begin{tabular}{|c|c|c|c|c|}
\hline order & $\begin{array}{c}\text { Super- } \\
\text { model } \\
\text { frequency } \\
/ \mathrm{Hz}\end{array}$ & $\begin{array}{c}\text { Simplified } \\
\text { model } \\
\text { frequency } / \mathrm{Hz}\end{array}$ & $\begin{array}{c}\text { Frequency } \\
\text { error } / \%\end{array}$ & $\begin{array}{c}\text { MAC } \\
\text { value }\end{array}$ \\
\hline 1 & 252.25 & 262.12 & 3.91 & 0.99 \\
\hline 2 & 321.39 & 326.98 & 1.74 & 0.99 \\
\hline 3 & 461.31 & 473.85 & 2.72 & 0.99 \\
\hline 4 & 590.65 & 606.18 & 2.63 & 0.98 \\
\hline 5 & 810.92 & 837.54 & 3.28 & 0.98 \\
\hline 6 & 909.8 & 934.19 & 2.68 & 0.99 \\
\hline 7 & 961.72 & 986.51 & 2.58 & 0.90 \\
\hline 8 & 1000.1 & 1015 & 1.49 & 0.92 \\
\hline 9 & 1059.5 & 1072.5 & 1.23 & 0.96 \\
\hline 10 & 1193.5 & 1221.9 & 2.38 & 0.98 \\
\hline
\end{tabular}

Table 2. Comparison of computational scale between the simplified model and super-model.

\begin{tabular}{|c|c|c|c|}
\hline & $\begin{array}{c}\text { Super- } \\
\text { model }\end{array}$ & $\begin{array}{c}\text { Simplified } \\
\text { model }\end{array}$ & $\begin{array}{c}\text { percentage } \\
/ \%\end{array}$ \\
\hline DOF & 979248 & 109647 & 11.20 \\
\hline $\begin{array}{c}\text { Calculation } \\
\text { time }\end{array}$ & 472.10 & 48.80 & 10.34 \\
\hline
\end{tabular}

\subsection{Model updating for the simplified model}

Some errors may exist in dynamic characteristics between the initial simplified model and the super-model. The geometric simplification and mesh discretization are the primary contributors to the inaccuracy of the initial simplified model. These errors can be reduced or corrected by the model updating procedure. Therefore, the initial simplified model can be updated to produce the dynamic characteristics in agreement with that of the super-model. It is noted from table 1 that the relative frequency errors between the initial simplified model and the super-model are large after simplification. Therefore, the modal frequencies of the initial simplified model can be corrected based on the results of the super-model. The elastic modulus of the five regions are selected as updating parameters, as shown in Figure 6(b), corresponding to the modified variables E1 to E5, respectively. The initial values are all set as $210 \mathrm{GPa}$. The updating parameters of the base are optimized using the sensitivity-based optimization. The updating parameters tend to converge after 16 iterations, as shown in Figure 7. After updating, the elastic modulus E1 to E5 in the updating regions of the base are $191 \mathrm{GPa}, 601 \mathrm{GPa}$ $175 \mathrm{GPa}, 600 \mathrm{GPa}$, and $552 \mathrm{GPa}$, respectively. It is noted that all the updating parameters have changed to some extent, which indicates that the stiffness of the simplified model is changed after simplification. The stiffness of the simplified model is compensated by modifying the elastic modulus, which makes the dynamic characteristics of the simplified model to be consistent with that of the reference model. Figure 8 gives the frequency comparison of the updated model with the super-model. From figure 8, we can see that the frequency errors of the updated model and the super-model decrease in relation to the initial simplified model. The maximum frequency error is reduced from $3.91 \%$ to $0.97 \%$ after updating, and the average frequency error is reduced from $2.46 \%$ to $0.59 \%$ after updating. Figures 9 gives the MAC comparison of the eigenvectors of the updated model with the super-model before and after updating. From Figure 9, it can be seen that the correlation of modal shapes between the updated simplified model and the super-model is very good after updating. We can see that the MAC values of the matched modal pairs are all greater than 0.9 . So the dynamic characteristics of the updated model are agree well with that of the supermodel, and the predictive accuracy of the updated model is significantly improved.

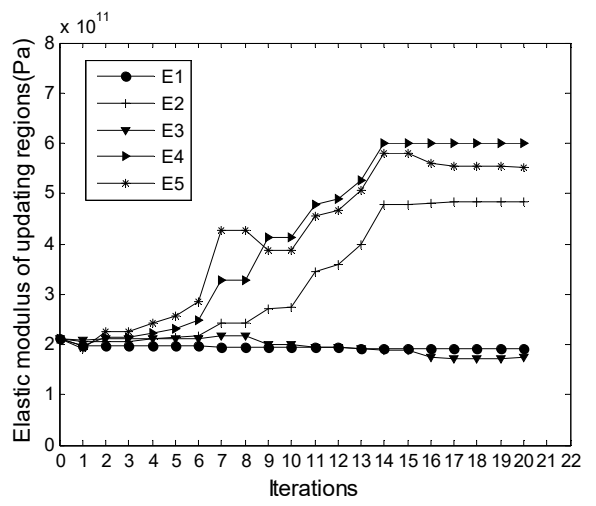

Fig. 7. Convergence of the updating parameters. 


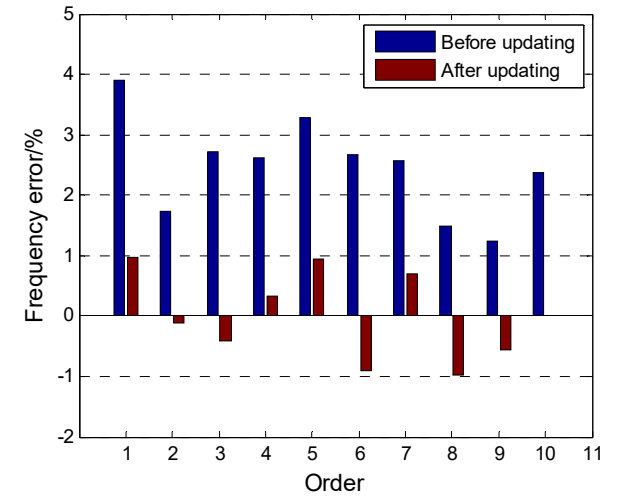

Fig. 8. Comparison of frequency errors before and after updating.

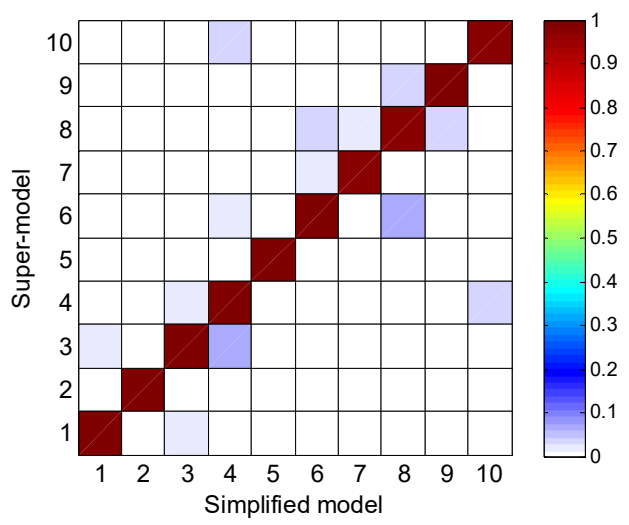

Fig. 9. MAC comparison of the simplified model with the super-model after updating.

\section{Dynamic prediction for the updated model}

\subsection{Dynamic prediction for the updated component}

The unbalance responses of the updated model and the super-model are predicted using harmonic analysis. The results are compared to further validate the effectiveness of the updating method. Figure 10 shows the excitation, response and constraint positions of the base FE model. Figures 11 respectively compares the frequencyamplitude curve for the super, initial and updated models, with a frequency range up to $1000 \mathrm{~Hz}$. The frequencyamplitude curve of the updated model is much closer to that of the super-model than that of the initial simplified model, which indicates that the updated model can accurately represents the dynamic characteristics of the super-model. So the updated model is valid within in the range of interest.

\subsection{Dynamic prediction for OLTC}

The whole FE model of OLTC is constructed based on the parts obtained by the updating method. The connections such as bolts and screws of the assembly model are modeled using rigid connection, but the springs are created by one-dimensional linear spring elements. Figure 12 shows the FE model of OLTC. The degree of freedom of the simplified FE model of OLTC is about one-tenth of the super-model, so the computation efficiency is improved a lot.

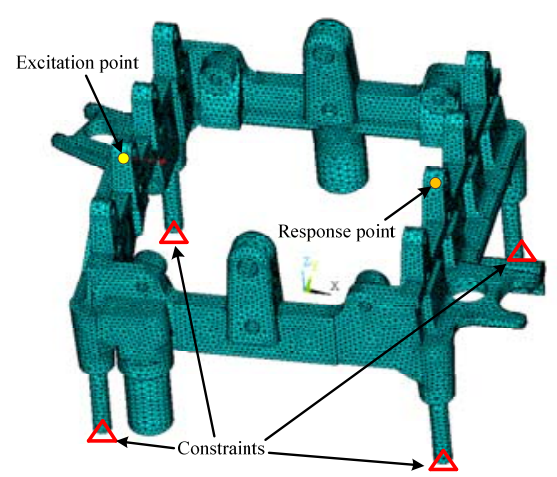

Fig. 10. Excitation, response and constraint positions of the base FE model.

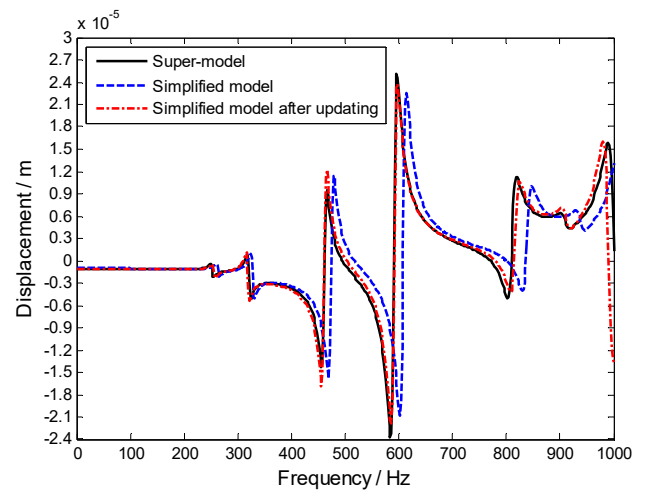

Fig. 11. Comparison of frequency-amplitude curves before and after updating.

The first 8 modal frequencies and mode shapes, as shown in Figure 13, are obtained by modal analysis of the simplified model of OLTC. From Figure 13, it is noted that OLTC has 8 vibration modes within the range of $151.2 \mathrm{~Hz}$, and most of the mode shapes show that the deformation of the hanging board is larger than that of other components, and the mode shapes of the hanging board mainly represent bending or torsional modes within $151.2 \mathrm{~Hz}$. Therefore, the hanging board is more sensitive to the excitation of low frequencies.

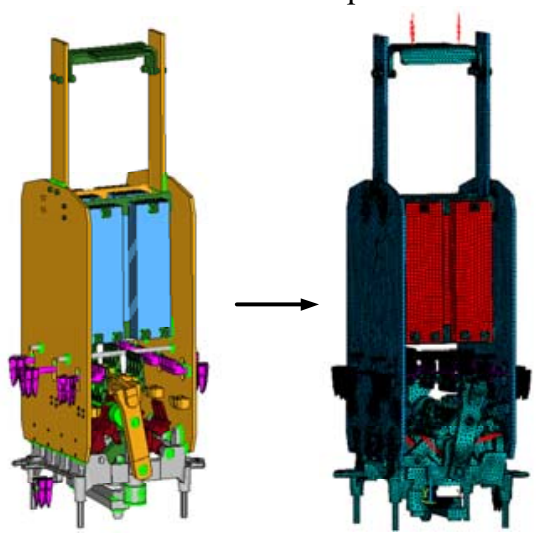

Fig. 12. Finite element model of assembled OLTC. 


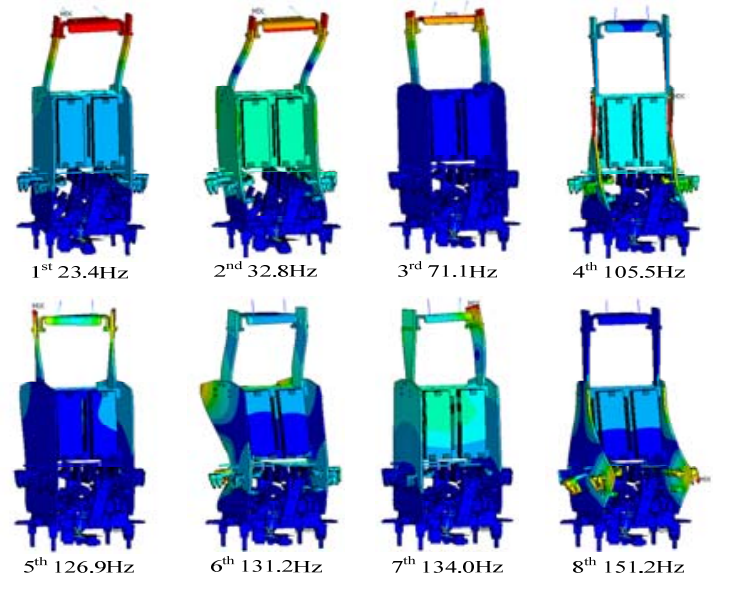

Fig. 13. Model shapes of OLTC.

\section{Conclusion}

A more precise FE model of a structure is extremely important for the dynamic prediction or for the structure fault diagnosis. This paper presents a method for dynamic model updating based on the super-model. Taking the modeling of OLTC base as an example, the updated simplified model of the base is obtained using sensitivity-based updating methodology based on the simulation data of the super-model. The maximum frequency error and the average frequency error of the first 10 modes between the simplified model and the super-model are $0.97 \%$ and $0.59 \%$, respectively, and the MAC values are all above 0.9 . However, the freedom and computation time of the simplified model are only $11.2 \%$ and $10.34 \%$ of the super-model, respectively. The main conclusions are as follows:

(1) The errors of the simplified FE model mainly consists of geometric simplification and mesh discretization. On the basis of shape feature, the elastic modulus of the blocks selected as updating parameters are feasible, and the results are good after updating. Actually, the errors of the simplified model are composed of mass and stiffness from the physical meaning. The precision of the updated model can be improved more if density and elastic modulus are selected to update simultaneously.

(2) The simplified model satisfying the convergence condition can be quickly obtained by the sensitivity-base updating method. However, the optimal solution may not easy to be found, and it tend to fall into local optimal solution. At this point, the initial updating parameters and constraint range of the state variables should be appropriately adjusted to get the optimal solution according to the influence of simplification on the dynamic characteristics of the FE model.

(3) The simulation results of the super-model can be guided to update the simplified model, but they are only the design expectations. The uncertainties of material physical parameters, manufacturing errors, assembly tolerance, boundary conditions are not considered compared with the actual structure, so these factors should be further taken into account on the effect of the updated results.

\section{Acknowledgments}

The financial support of University science research project of Guangxi zhuang autonomous region of China (No. KY2015YB344) is gratefully acknowledged.

\section{Renference}

1. Sponsor. IEEE Standard Requirements for Load Tap Changers. IEEE Std C57, 1-46(1995)

2. R. Bhuyan. Acoustic diagnostic of On-Load Tap Changers (OLTC) and Investigation of the Arcing Phenomenon. (2013)

3. C. Bengtsson. Status and trends in transformer monitoring. IEEE Transactions on Power Delivery, 11, 1379-1384(1996)

4. A. Cichoń, S. Borucki, P. Berger. Selecting Sensor for On Load Tap Changer Contacts Degree of Wear Diagnostics. Acta Physica Polonica, 124, 395-398 (2013)

5. J. Seo, H. Ma, T. K. Saha. Vibration measurement and signal processing for condition assessment of OLTC of transformer. Power and Energy Engineering Conference, IEEE, 1-5(2015)

6. J. Seo, H. Ma, T. Saha. On Savitzky-Golay Filtering for Online Condition Monitoring of Transformer OnLoad Tap Changer. IEEE Transactions on Power Delivery, 1,99(2017)

7. P. Kang, D. Birtwhistle. Characterisation of Vibration Signals Using Continuous Wavelet Transform for Condition Assessment of On-load Tap Changers. Mechanical Systems and Signal Processing, 17, 561-577(2003)

8. G. M. Mikheev, T. G. Ivanova, K. U. Kalandarov, et al. Diagnostics of Transformer Switching Devices. Power Technology \& Engineering, 50, 1-7(2016)

9. Q. Li, T. Zhao, L. Zhang, et al. Mechanical Fault Diagnostics of Onload Tap Changer Within Power Transformers Based on Hidden Markov Model. IEEE Transactions on Power Delivery, 27, 596601(2012)

10. J. E. Mottershead, M. I. Friswell. Model Updating In Structural Dynamics: A Survey. Journal of Sound \& Vibration, 167, 347-375(1993)

11. C. Zang, D.J. Ewins. Model validation of structural dynamics in the aero-engine degine process. Front. Energy Power Eng. China, 3, 480-488(2009)

12. W. F. Daniel. Development of valid FE Models for Structural Dynamic Design, Imperil college, (2001)

13. J. E. Mottershead, M. Link , M. I. Friswell, The sensitivity Method in Finite Element Modal Updating : A Tutorial. Mechanical System and Signal Processing, 25, 2275-2296(2011)

14. R. J. Allemang. The Modal Assurance Criterion Twenty Years of Use and Abuse. Spie Proceedings, , 37, 14-23(2003) 